different strain mapping techniques are discussed.

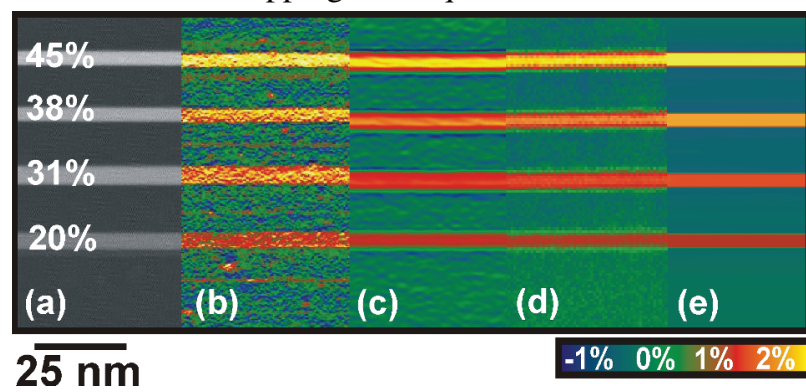

Figure 1. HAADF STEM image of the calibration specimens with deformation maps acquired by HAADF STEM, dark holography, precession diffraction and finite element simulations.

Keywords: Strain Mapping, TEM, Semiconductors.

\section{MS27-O2 Electron diffraction and imaging of 3D nanocrystals of pharmaceuticals, peptides and proteins}

Jan Pieter Abrahams ${ }^{1,2,3}$, Eric van Genderen ${ }^{1}$, Igor Nederlof ${ }^{4}$, Max Clabbers ${ }^{1}$, Yaowang $\mathrm{Li}^{1}$

1. LIC, Leiden University, Einsteinweg 55, 2333CC Leiden, The Netherlands

2. Biozentrum, University of Basel, Klingelbergstrasse 50/70 CH 4056 Basel Switzerland

3. LBR, Paul Scherrer Institut, 5232 Villigen PSI, Switzerland

4. Amsterdam Scientific Instruments, Science Park 1051098 XG

Amsterdam The Netherlands

email: Abrahams@chem.leidenuniv.nl

High-energy electrons provide 1000 times more information per Gray (absorbed energy, i.e. radiation damage), compared to X-rays. Hence electrons outperform X-rays for structure determination when radiation damage is the limiting factor. Unlike X-rays, electrons can not only be diffracted, but also imaged. However, when imaging transparent samples, the total number of quanta per unit area determines the signal-to-noise ratio. When diffracting transparent samples, the number of interacting quanta per unit area determines the signal-to-noise ratio. Measuring electron diffraction accurately has only recently become possible with the advent of quantum area detectors. One of the challenges is that an electron microscope is flooded with photon radiation resulting from Bremsstrahlung generated by the high-energy electrons. Only an area detector that can discriminate between photon noise and electron signal is insensitive to this noise.

This difference in signal-to-noise ratio was demonstrated in practice for electrons using a Timepix quantum area detector. When imaging a $100 \mathrm{~nm}$ thick lysozyme protein crystal with electrons, typically one to two images of the same location could be measured with significant details up to $3.5 \AA$ resolution. Subsequent images had suffered too much from radiation damage to show such detail. When diffracting similar crystals, hundreds of electron diffraction patterns with Bragg spots beyond $3 \AA$ resolution could be measured from the same location. However, there is no such thing as a free lunch. Diffraction comes at a price: the structure factor phases are lost. They can only be retrieved using additional (prior) information, for instance obtained from (a few) electron images. We demonstrated this strategy by phasing the $3 \mathrm{D}$ structure factors of a nano-crystalline amyloidic peptide.

Keywords: electron diffraction, phasing, detectors 